\title{
Up-to-date results and upgrade plans of the MEG experiment
}

\author{
Hajime Nishiguchi* \\ KEK - High Energy Accelerator Research Organization, 1-1 Oho, Tsukuba, 305-0801, Japan \\ E-mail: hajime.nishiquchi@kek.ip
}

\section{Giovanni Signorelli}

INFN Sezione di Pisa, Largo B. Pontecorvo 3, 56127 Pisa, Italy

The MEG experiment at the Paul Scherrer Institut (Switzerland) aims at searching for the LeptonFlavour Violating (LFV) muon decay, $\mu^{+} \rightarrow \mathrm{e}^{+} \gamma$, with unprecedented sensitivity. Such decay is forbidden within the Standard Model, nevertheless all its viable extensions predict a branching ratio for this decay in the $10^{-14}$ to $10^{-12}$ range. Data collected in 2009 and 2010, which corresponds to a total of $1.8 \times 10^{14}$ muon decays, allowed us to set the most stringent limit to date on charged $\operatorname{LFV}\left(\mathcal{B R}\left(\mu^{+} \rightarrow \mathrm{e}^{+} \gamma\right)<2.4 \times 10^{-12}\right.$ at $90 \%$ C.L. $)$. The status of the experiment during the last data taking is presented together with preliminary analysis of 2011 data.

In parallel with the data-taking, the MEG collaboration has recently started to discuss the upgrade plan in order to perform the experiment with higher beam intensity and better measurement resolutions, and already started several associated $R \& D$. In addition to the future prospects, the detailed ideas of upgrade are also presented.

36th International Conference on High Energy Physics,

July 4-11, 2012

Melbourne, Australia

${ }^{*}$ Speaker. 


\section{Introduction}

A Lepton Flavour Violation (LFV) among charged leptons, e.g. $\mu^{+} \rightarrow \mathrm{e}^{+} \gamma$ decay etc., which has never been observed while the quark mixing and the neutrino oscillations have been experimentally confirmed, is attracting a great deal of attention, since its observation is highly expected by many of well motivated theories beyond the Standard Model[四]. It is predicted that $\mu^{+} \rightarrow \mathrm{e}^{+} \gamma$ is naturally causable with a branching ratio just below the current upper bound, $10^{-11} \sim 10^{-14}$, by the leading theories for physics beyond the standard model, e.g. the Supersymmetric (SUSY) theories of Grand Unification (GUT) or Supersymmetric Standard Model with the seesaw mechanism (e.g. see Ref.[[]] for a review). According to such theories we should have observed several low mass states. However, these have not been discovered yet in energy frontier experiments and this represents one of the aims, unfortunately not yet reached, of the LHC program. Experiments searching for charged LFV processes can provide another approach to clarify these theories since they are very sensitive to SUSY and particularly to SUSY-GUT models. In this sense, charged LFV experiments are complementary to LHC in testing these theories.

The ambitious goal of the MEG experiment [3] is to search for a $\mu^{+} \rightarrow \mathrm{e}^{+} \gamma$ decay with an improved sensitivity by at least two orders of magnitude over the last best upper limit on a $\mu^{+} \rightarrow \mathrm{e}^{+} \gamma$ branching ratio, $1.2 \times 10^{-11}$ [䧃]. The signal of $\mu^{+} \rightarrow \mathrm{e}^{+} \gamma$ decay is very simple and is characterized by a 2-body final state of a positron and $\gamma$-ray pair emitted in opposite directions with the same energy, $52.8 \mathrm{MeV}$, which corresponds to half the muon mass. There are two major backgrounds in the search for $\mu^{+} \rightarrow \mathrm{e}^{+} \gamma$. One is a physics (prompt) background from a radiative muon decay, $\mu^{+} \rightarrow \mathrm{e}^{+} \nu_{\mathrm{e}} \overline{\nu_{\mu}} \gamma$, when the positron and the $\gamma$-ray are emitted back-to-back with the two neutrinos carrying off tiny energy. The other background is an accidental coincidence of a positron from a normal Michel decay, $\mu^{+} \rightarrow \mathrm{e}^{+} \nu_{\mathrm{e}} \overline{\nu_{\mu}}$, with a high energy random photon. The source of high energy $\gamma$ ray is either a radiative muon decay, annihilation-in-flight or external bremsstrahlung of a positron. The background is primarily dominated by this accidental coincidence. Suppressing such an accidental overlap thus holds the key for leading MEG to a successful conclusion.

\section{The MEG Experiment}

A schematic view of the MEG detector apparatus is shown in Figure $\mathbb{W}$. A more detailed



A DC muon beam is the best tool to search for $\mu^{+} \rightarrow \mathrm{e}^{+} \gamma$ since experimental sensitivity is mainly limited by accidental backgrounds. The MEG experiment thus employs using the world's most intense DC muon beam which is available at the Paul Scherrer Institut (PSI).

The momentum and direction of positrons are measured precisely by a Positron Spectrometer, which consists of a superconducting solenoidal magnet specially designed to form a highly graded magnetic field, an ultimate low-mass drift chamber system, and a precise time measuring counter system [ [6]. The MEG solenoidal magnet provides a highly graded magnetic field (1.27 $\mathrm{T}$ at the centre and decreasing down to $0.49 \mathrm{~T}$ at the end along the beam axis). Thanks to this graded magnetic field, the MEG solenoid can sweep positrons out of the fiducial tracking volume quickly while positrons undergo many turns in the tracker volume in a uniform solenoidal field. Additionally, positrons with the same absolute momenta follow trajectories with a constant pro- 

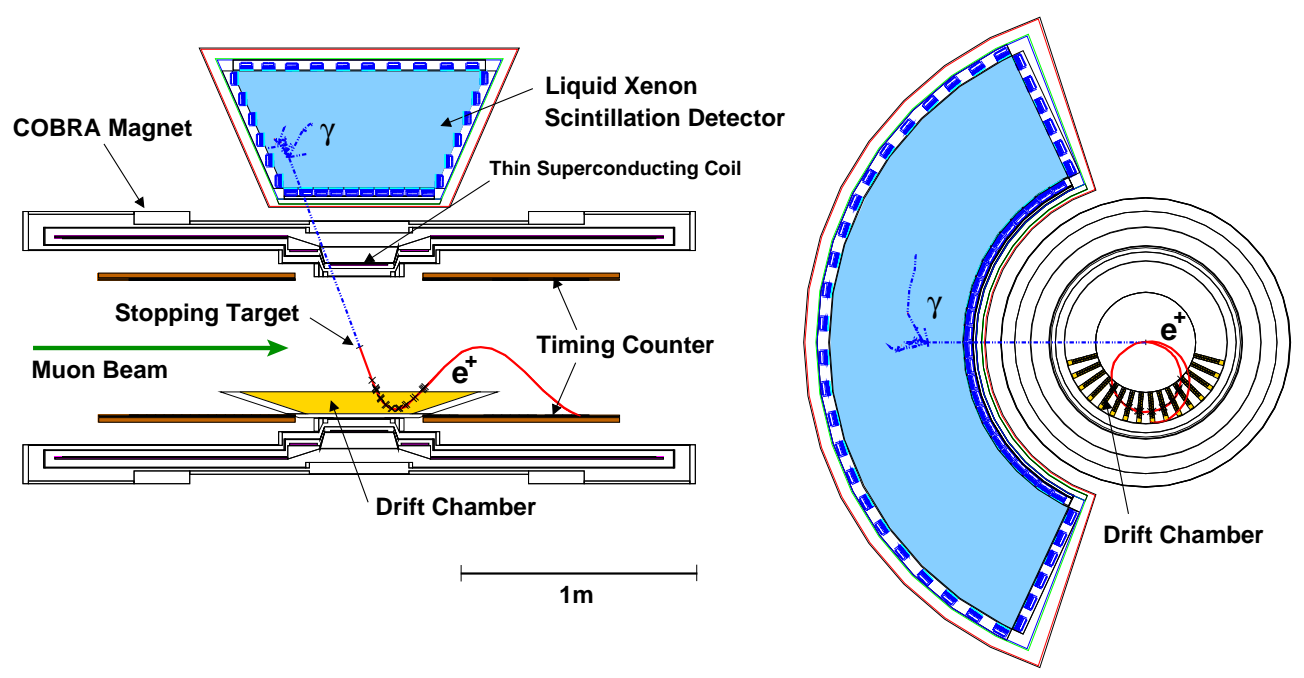

Figure 1: A schematic view of the MEG detector apparatus

jected bending radius independent of the emission angles, while in a uniform solenoidal field the bending radius depends on the emission angle. This allows us to discriminate sharply high momentum signal positrons from the tremendous Michel positron background originating from the muon-stopping target. The Positron Spectrometer therefore does not need to measure the positron trajectory in the small radius region, i.e. the drift chambers can be blind to most of the Michel positrons.

While all positrons are confined by the solenoid, the $\gamma$ ray pass through the thin superconducting coil of the spectrometer with $\approx 80 \%$ transmission probability, and are detected by an innovative liquid-xenon photon detector $[\square]$. Scintillation light emitted inside liquid xenon are viewed from all sides by photo-multiplier tubes (PMT) that are immersed in liquid xenon in order to maximize direct light collection. Liquid-xenon scintillator has very high light yield ( $\approx 75 \%$ of $\mathrm{NaI}$ crystal) and fast response, which are the most essential ingredients for precise energy and timing resolutions required for this experiment.

\section{MEG Physics Run 2009, 2010 and 2011}

The results based on data collected in 2009 and $2010^{1}$ (for a total of $1.8 \times 10^{14} \mu^{+}$decays in the target) could give a $90 \%$ C.L. upper limit of $2.4 \times 10^{-12}$ on $\mathcal{B R}\left(\mu^{+} \rightarrow \mathrm{e}^{+} \gamma\right)$, constituting the most stringent limit on the existence of this decay to date [ [8].

We adopted a likelihood analysis method combined with a blind procedure on examining the data: events close to the signal region were kept hidden (blind region) until all the analysis procedures had been completely defined. The probability density functions (PDFs) needed for the likelihood analysis were constructed using the events outside of the blind region (sidebands). The kinematic variables used to identify the $\mu^{+} \rightarrow \mathrm{e}^{+} \gamma$ decays are the $\gamma$-ray and $\mathrm{e}^{+}$energies $\left(E_{\gamma}, E_{\mathrm{e}}\right)$, their relative directions $\left(\theta_{\mathrm{e} \gamma}, \phi_{\mathrm{e} \gamma}\right)$, and emission time $\left(t_{\mathrm{e} \gamma}\right)$. The off-line event selection requires at

\footnotetext{
${ }^{1}$ The 2010 statistics are approximately twice that of 2009.
} 
Table 1: Experiment parameters in 2009, 2010 and 2011

\begin{tabular}{lccc}
\hline performances & 2009 & 2010 & 2011(preliminary) \\
\hline$\sigma_{E_{\gamma}} / E_{\gamma}(\%)($ conversion depth $>2 \mathrm{~cm})$ & 1.9 & $\leftarrow$ & 1.7 \\
$\sigma_{x_{\gamma}}(\mathrm{mm})$ & $5-6$ & $\leftarrow$ & $\leftarrow$ \\
$\epsilon_{\gamma}(\%)$ & 59 & $\leftarrow$ & 63 \\
\hline$\sigma_{E_{\mathrm{e}}} / E_{\mathrm{e}}(\%)($ core fraction $(\%))$ & $0.59(80 \%)$ & $0.61(79 \%)$ & $0.61(86 \%)$ \\
$\sigma_{\theta_{\mathrm{e}}}, \sigma_{\mathrm{e} \gamma}(\mathrm{mrad})$ & $9.4,6.7$ & $11.0,7.2$ & $10.8,6.5$ \\
$\epsilon_{\mathrm{e}}(\%)$ & 40 & 41 & $\leftarrow$ \\
\hline$\sigma_{t_{\mathrm{e} \gamma}}(\mathrm{ps})$ & $146(\mathrm{core})$ & $126($ core $)$ & 133 \\
$\mu^{+}$decay vertex resolution $(\mathrm{mm})$ & $1.1-1.5$ & $1.1-2.0$ & $1.0-1.9$ \\
$\epsilon_{\text {trig }}(\%)$ & 91 & 92 & 95 \\
\hline Number of stopped $\mu^{+}$on the target & $7.0 \times 10^{13}$ & $1.1 \times 10^{14}$ & $1.9 \times 10^{14}$ \\
\hline \hline
\end{tabular}

least one $\mathrm{e}^{+}$track reconstructed in the spectrometer and pointing to the target, with minimal quality cuts applied.

Detector performances are listed in Table $\square$ that is used as parameters in order to carry out the physics analysis; analysis-region determination, PDFs construction, background estimation etc., where the values for 2011 are preliminary estimation.

A likelihood analysis is carried out for events in a portion of the blind region (analysis region) defined by $48<E_{\gamma}<58 \mathrm{MeV}, 50<E_{\mathrm{e}}<56 \mathrm{MeV}$, $\left|t_{\mathrm{e} \gamma}\right|<0.7 \mathrm{~ns},\left|\theta_{\mathrm{e} \gamma}\right|<50 \mathrm{mrad}$, and $\left|\phi_{\mathrm{e} \gamma}\right|<$ $50 \mathrm{mrad}$. These intervals in the analysis variables are between $5 \sigma$ and $20 \sigma$ wide to fully contain the signal events and also retain some background events. The best estimates of the numbers of signal, radiative muon decay (RMD), and accidental background (BG) events in the analysis region are obtained by maximizing the following likelihood function:

$$
\begin{aligned}
\mathcal{L}\left(N_{\mathrm{sig}}, N_{\mathrm{RMD}}, N_{\mathrm{BG}}\right)= & \frac{\mathrm{e}^{-N}}{N_{\mathrm{obs}} !} \mathrm{e}^{-\left[\left(N_{\mathrm{RMD}}-\left\langle N_{\mathrm{RMD}}\right\rangle\right)^{2} / 2 \sigma_{\mathrm{RMD}}^{2}\right]} \times \mathrm{e}^{-\left[\left(N_{\mathrm{BG}}-\left\langle N_{\mathrm{BG}}\right\rangle\right)^{2} / 2 \sigma_{\mathrm{BG}}^{2}\right]} \\
& \times \prod_{i=1}^{N_{\mathrm{obs}}}\left(N_{\mathrm{sig}} \cdot S\left(\mathbf{x}_{i}\right)+N_{\mathrm{RMD}} \cdot R\left(\mathbf{x}_{i}\right)+N_{\mathrm{BG}} \cdot B\left(\mathbf{x}_{i}\right)\right)
\end{aligned}
$$

where $\mathbf{x}_{i}=\left(E_{\gamma}, E_{\mathrm{e}}, t_{\mathrm{e} \gamma}, \theta_{\mathrm{e} \gamma}, \phi_{\mathrm{e} \gamma}\right)$ is the vector of observables for the $i$-th event, $N_{\mathrm{sig}}, N_{\mathrm{RMD}}$, and $N_{\mathrm{BG}}$ are the expected numbers of signal, RMD and BG events, while $S, R$ and $B$ are their corresponding PDFs. $N\left(=N_{\text {sig }}+N_{\mathrm{RMD}}+N_{\mathrm{BG}}\right)$ is the observed total number of events in the analysis window. Figure $\square$ shows 2009 and 2010 combined data in the analysis box and the fitting results. No significant excess was found in the dataset; and the upper limit of the branching ratio is set to be $2.4 \times 10^{-12}$ at $90 \%$ C.L. The upper limit is about 5 times tighter than the previous upper limit and the new physics are constrained stringently.

The physics-data-taking for 2011 was also successfully completed doubling the total data statistics, with the analysis currently being pursued. 

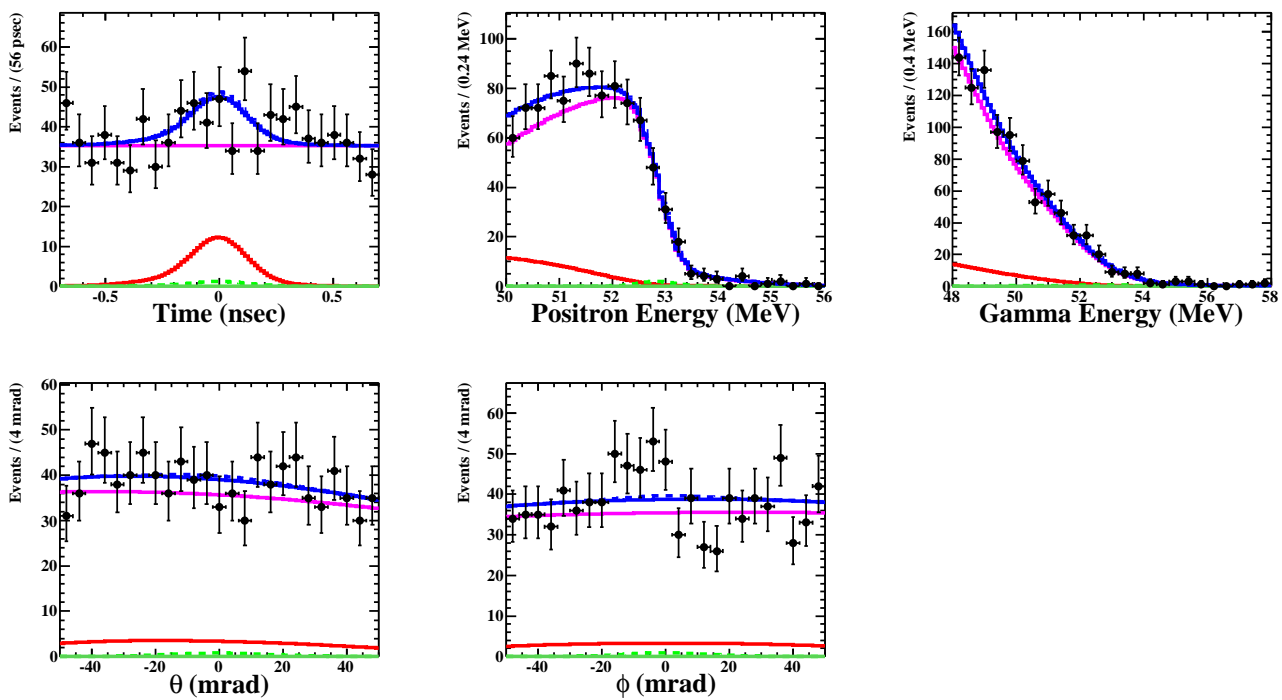

Figure 2: Event distribution in the fit region projected on each variable $\left(t_{\mathrm{e} \gamma}, E_{\mathrm{e}}, E_{\gamma}, \theta_{\mathrm{e} \gamma}, \phi_{\mathrm{e} \gamma}\right)$ and result of the maximum likelihood fit. The green, red and magenta curves show signal, RD, and BG PDFs, respectively, and blue for the total. The solid lines show the best-fit distributions, while the dashed ones show those with $N_{\text {sig }}$ at the $90 \%$ upper limit.

\section{Current Status and Upgrade Plans}

The MEG experiment is expected to continue data-taking until mid 2013, when a sensitivity of $\sim 6 \times 10^{-13}$ is expected to be reached, beyond which only limited improvement is expected due to the anticipated increasing dominance of background events in the signal region. Hence, in order to significantly improve the sensitivity reach with a goal of being able to detect the $\mu^{+} \rightarrow \mathrm{e}^{+} \gamma$ decay at a level of about one order of magnitude better, a new upgraded MEG experiment is required. The MEG collaboration thus has been making intensive efforts for the upgrade R\&D recently.

The key features of this new MEG upgrade are to increase the rate capability of all detectors to enable running at the intensity frontier, while also improving the energy, angular and timing resolutions, for both the positron and photon arms of the MEG detector. For instance, the liquidxenon photon detector [ []] will be improved by increasing the granularity at the incident face, by replacing the current PMTs with a larger number of smaller photosensors[Q] and optimizing the photosensor layout also on the lateral faces, as shown in Figure [3. This should lead to improved energy and spatial resolutions of the liquid-xenon photon detector. On the other hand, positron spectrometer will also be improved by a new low-mass, single volume, high granularity tracker. This, in combination with a thinner stopping target and hence a reduction in the multiple scattering of the positrons, will lead to the spatial, angular and energy requirements being met on the positron side. A new highly segmented and fast timing counter array will replace the current system, so allowing improved timing resolution capabilities in order to minimize the number of background events entering the signal-timing window.

Intensive R\&D studies are ongoing to realize this new MEG upgrade. An initial period of 

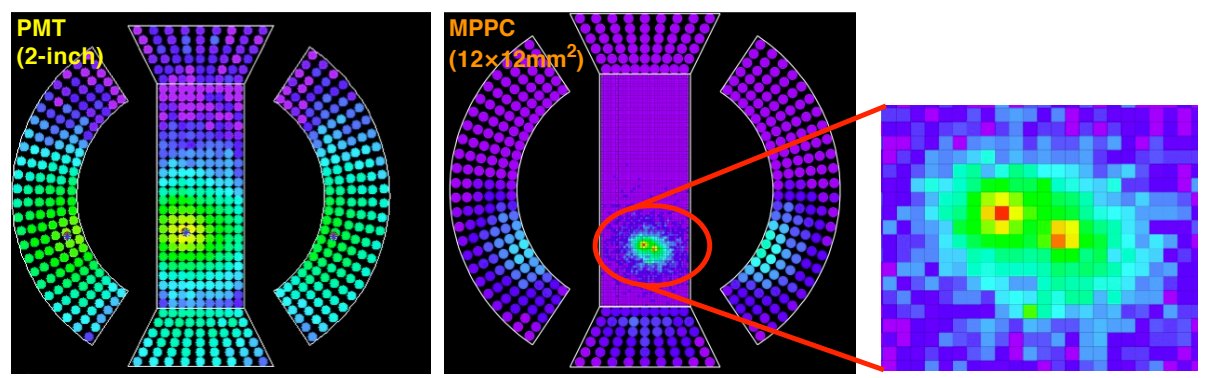

Figure 3: Typical examples of scintillator light distribution seen by photo-sensors in case of (left) PMTs and (right) smaller photo sensors $\left(12 \times 12 \mathrm{~mm}^{2}\right)$ on the $\gamma$ entrance face.

design and development for the upgrade, with the planned end of construction date of around mid 2015, is to be followed by an engineering run in the latter half of 2015 and, providing the performance is as expected, data-taking could start in 2016. With a muon stopping rate of $7 \times$ $10^{7} \mu^{+} / \mathrm{sec}$ and a target thickness of $140 \mu \mathrm{m}$ and assuming 180 DAQ-days per year, we expect to achieve a final sensitivity of about $6 \times 10^{-14}$ in 3 years of running.

\section{Conclusion}

The MEG experiment is searching for a charged LFV decay, $\mu^{+} \rightarrow \mathrm{e}^{+} \gamma$, which is clearly sensitive to new physics beyond the Standard Model. Data collected in 2009 and 2010 allowed us to set the most stringent limit to date on $\mathcal{B R}(\mu \rightarrow \mathrm{e} \gamma)$. Run 2011 was successfully completed doubling the total data statistics, and currently in parallel to data taking, the MEG collaboration is making efforts to upgrade the experiment aiming to reach unprecedented sensitivity of $6 \times 10^{-14}$.

\section{Acknowledgments}

We are grateful for the support and co-operation provided by PSI as the host laboratory and to the technical and engineering staffs of our institutes. This work is supported by MEXT KAKENHI 22000004 (Japan), INFN (Italy) and DOE DEFG02- 91ER40679 (USA).

\section{References}

[1] R. Barbieri and L.J. Hall, Phys. Lett. B338 (1994) pp.212-218

[2] Y. Kuno and Y. Okada, Rev. Mod. Phys. 73 (2001) pp.151-202

[3] MEG Collaboration, PSI Research Proposal R-99-05 (1999), (http://meg.psi.ch)

[4] M. L. Brooks et.al. [MEGA Collaboration], Phys. Rev. Lett. 83 (1999) pp.1521-1524

[5] J. Adam et.al. [MEG Collaboration], Nucl. Phys. B834 (2010) pp.1-12

[6] H. Nishiguchi, Nucl. Instr. Meth. A581 (2007) pp.538-541

[7] S. Mihara et.al., J. Phys.: Conf.Ser. 308 (2011) 012009

[8] J. Adam et.al. [MEG Collaboration], Phys. Rev. Lett. 107 (2011) 171801

[9] W. Ootani et.al., PoS(PhotoDet 2012) 035 